\title{
Design of the Temperature and humidity sensor and vibration sensor Interface Circuit for the Intelligent Managerial System of Blasting Equipment Library
}

\author{
Xingshan $\mathrm{Li}^{1, \text { a }}$, Yuanshen GUO ${ }^{2}$, Jian $\mathrm{LU}^{3}$ \\ ${ }^{1}$ Luohe Medical College, Luohe, 462000, China \\ ${ }^{2}$ Henan Quality Engineering Vocational College, Pingdingshan, 467000, China \\ ${ }^{3}$ Henan Industrial Vocational and Technical College, Nanyang, 473000, China \\ aemail:604141388@qq.com
}

\begin{abstract}
Keywords: Coal Mine Blasting Equipment Library; Intelligence; Single Chip Microcomputer (SCM); Temperature and humidity sensor ;vibration sensor; Module Interface Circuit.

Abstract. This paper mainly analyses the necessity to manage the explosive in the coal mine blasting equipment library. It makes intelligent management possible by using digital monitoring technology, communication technology and software technology and sensor technology for reference. High performance microcontroller was used for interface circuit design of temperature, humidity and vibration sensor, the results shown that normal operation. This study may represent a novel candidate resource for intelligent management of equipment library.
\end{abstract}

\section{Introduction}

In order to ensure the safety in the production of coal mine, the storage of powder and its access management must be strict and intelligent. With the rapid development of digital control technology, we can make intelligent management possible by using Temperature and humidity sensor and vibration sensor interface circuit.

\section{Component of Coal Mining Blasting Equipment Library System}

The intelligent management system is made up of three parts: the lower machine, the control device and PC. The system is shown as Fig.1.

\section{Control System of the Lower Machine}

The storage and access of the explosives and detonators in the reservoir area are managed by the lower machine (computer entrance guard system). The control system of lower machine is mainly composed of single chip microcomputer STC12C5A60S, temperature and humidity sensor, vibration sensor, the fingerprint machine, electromagnetic lock control and communication module [1]. The lower machine uses single chip to control the system [2]. The system is shown as Fig.2.

\section{Interface circuit of temperature, humidity and vibration sensor}

Temperature and humidity sensor is an equipment or a device which can convert the amount of temperature and humidity into electrical signal of easy to measure. Temperature and humidity sensor on the market in general is measuring the temperature and relative humidity.

Temperature and humidity of system were detected by capacitive digital temperature and humidity sensor AM2301 which has character of mature technology, stablity and reliability. In fact, there have a NTC temperature sensor, a capacitive humidity sensor and an 8-bit microcontroller in digital temperature and humidity sensor AM2301 ${ }^{[3]}$. Similar to the temperature sensor DS18B20, single wire transfer was used between single-chip microcomputer with the AM2301 interface, occupy less port, communication distance can be up to 20 meters and its data transmission formats: 
40 bit data $=16+16$ bit humidity data bit temperature data +8 bit checksum ${ }^{[4]}$.

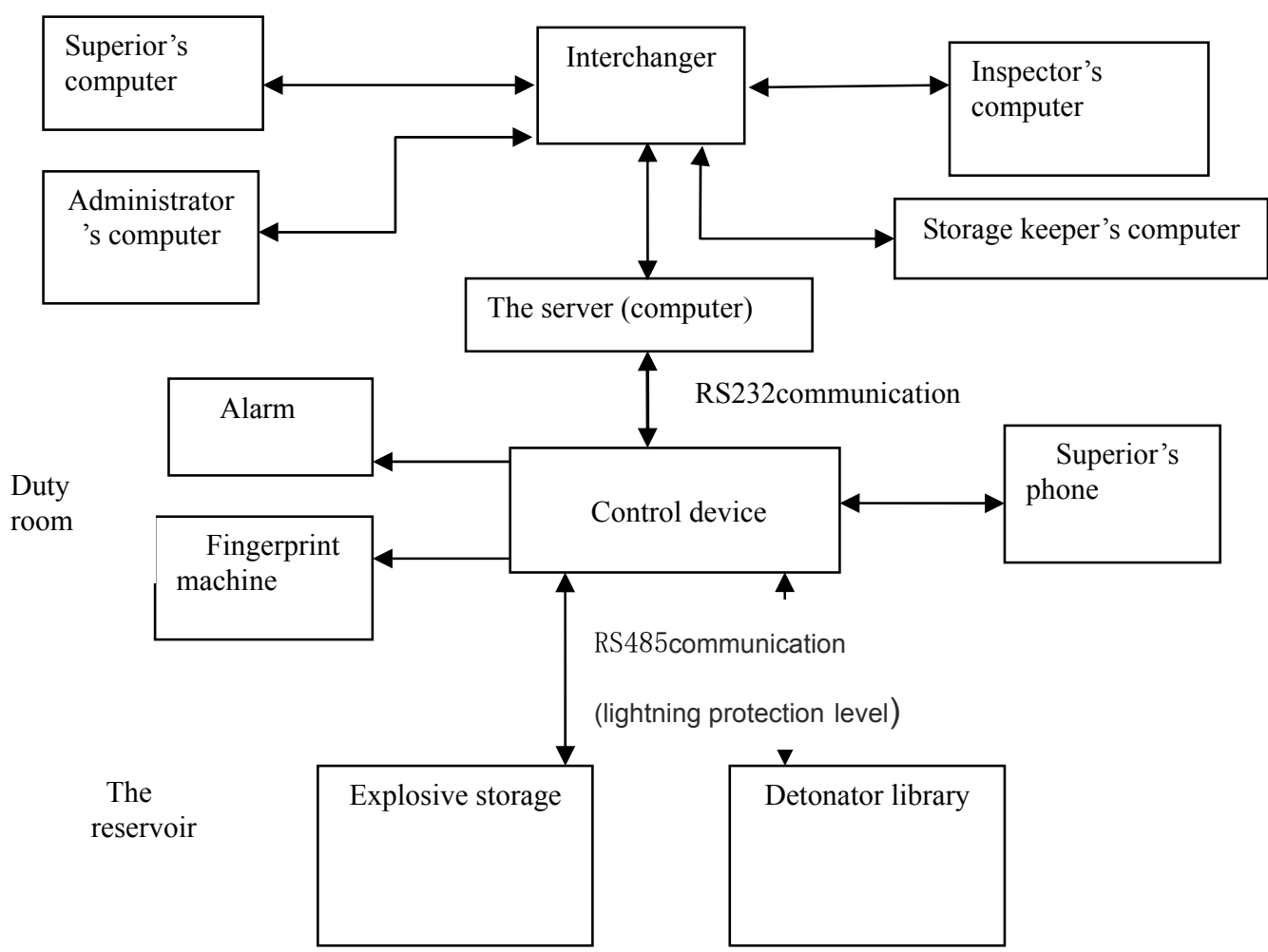

Fig.1. Managerial system of the coal mining blasting equipment library

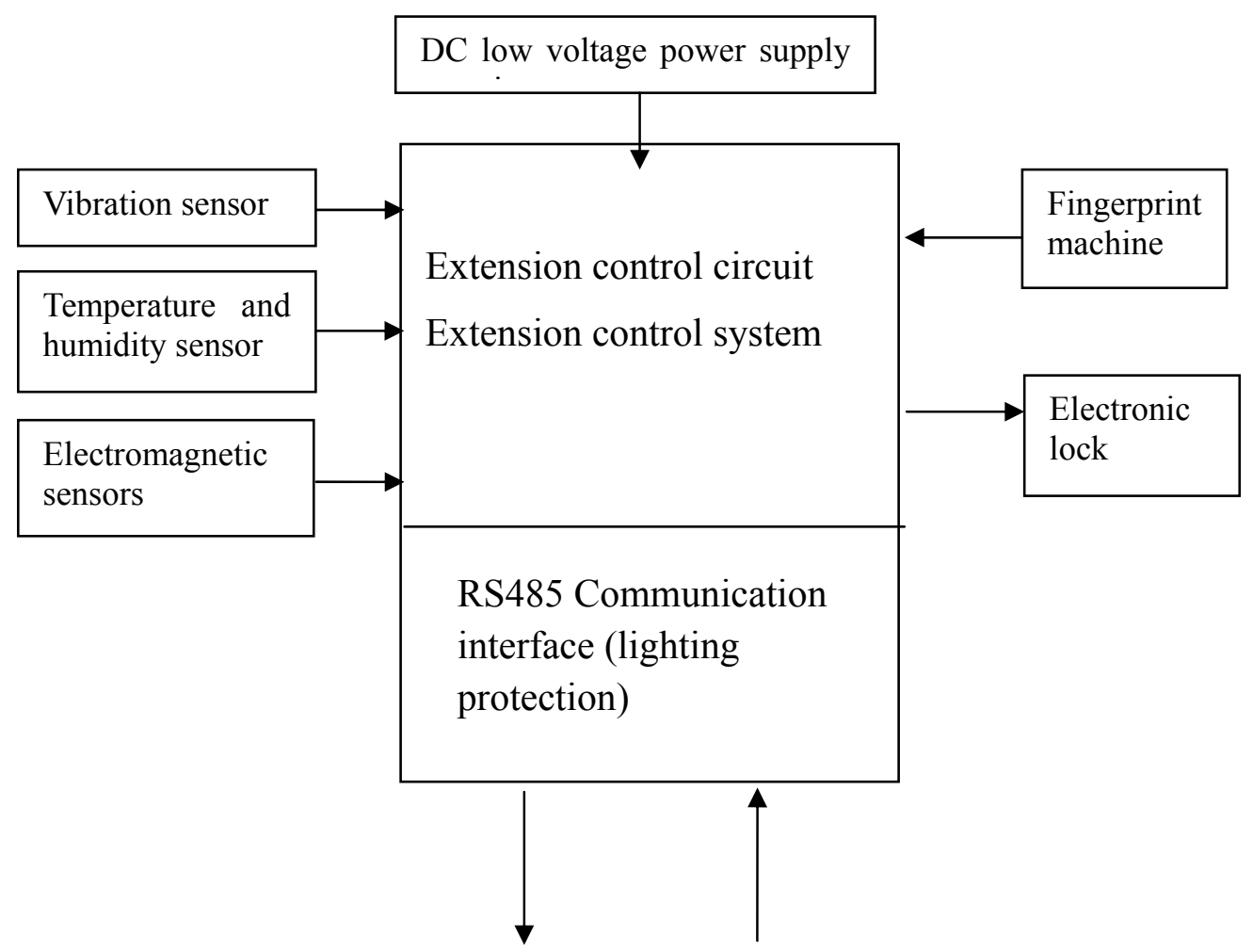

Fig.2. Control system of lower machine for coal mining blasting equipment library

Vibration sensor system is consist of buzzer and contact spring, vibration signal was tested by spring heavy hammer structure with piezoelectric ceramic chip, and through LM358 op-amp amplifier and output control signals, which has the advantages of low cost, high sensitivity, stable and reliable work and vibration testing wide adjustable. It was widely applied in anti-theft system of 
automobile and motorcycle. Vibration sensor was used in $80 \%$ vehicle alarm now. Sensors can also be with single chip microcomputer, wireless transmitting module, cable warning systems, application is very extensive.

Vibration sensor rated working voltage of $12 \mathrm{~V} \mathrm{DC}$, minimum working voltage must be greater than $5 \mathrm{~V}$, the output is detected a vibration output 1 second drop-down signals[5]. Sensor's red lines connect the $+12 \mathrm{~V}$, black lines connect GND, blue line is the signal output end of the sensor, detected a vibration output 1 second drop-down signals, the port can be direct and single chip microcomputer interface, no vibration at high level. Vibration detection sensitivity of the sensor is continuous adjustable, sensitivity increased at clockwise and lower sensitivity at counterclockwise.

Coal mine blasting equipment library temperature and humidity sensor, vibration sensor interface circuit (Figure 3)

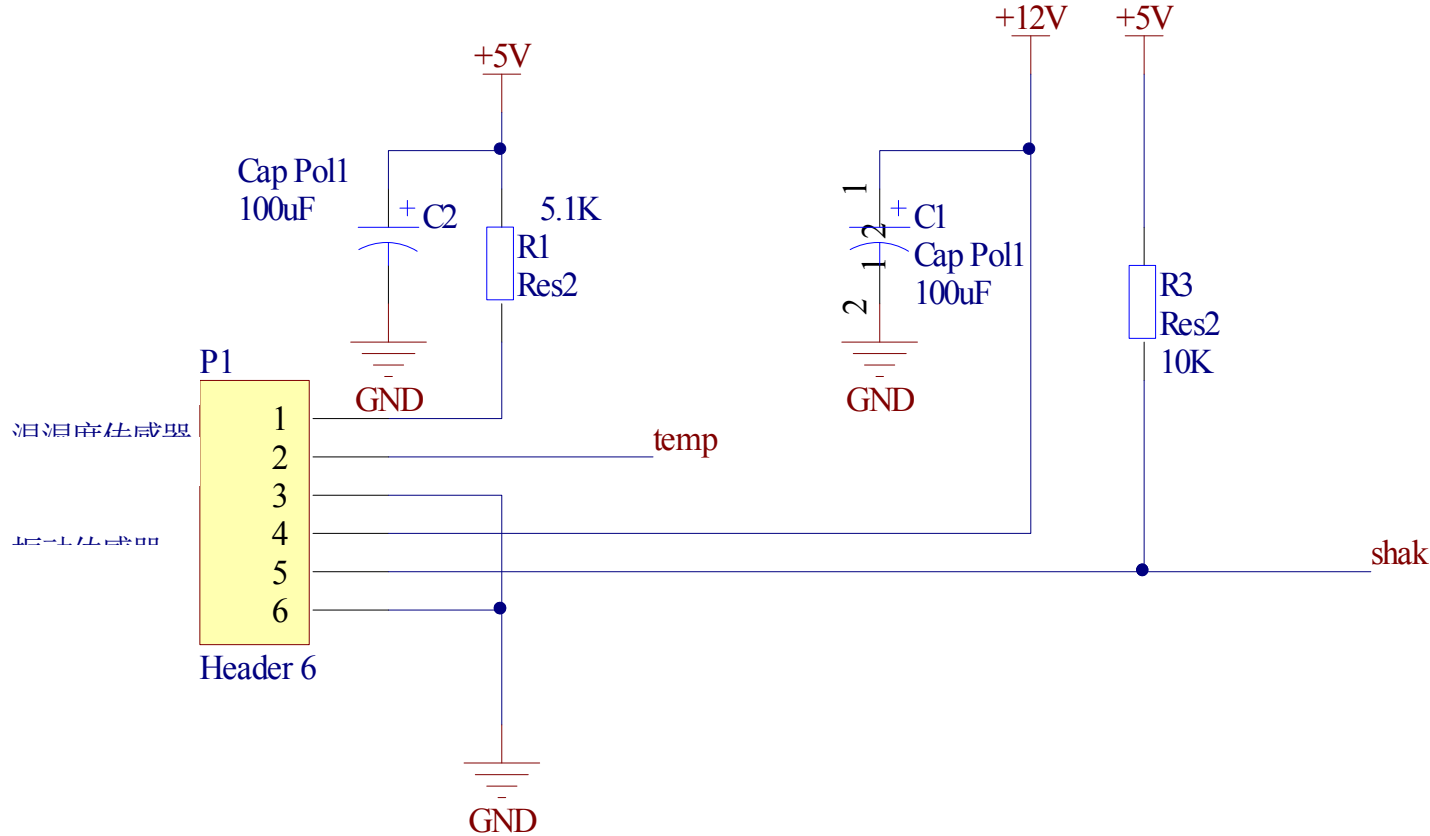

Fig.3. Temperature and humidity sensor and vibration sensor interface circuit

\section{Test results}

The program for Temperature and humidity sensor.

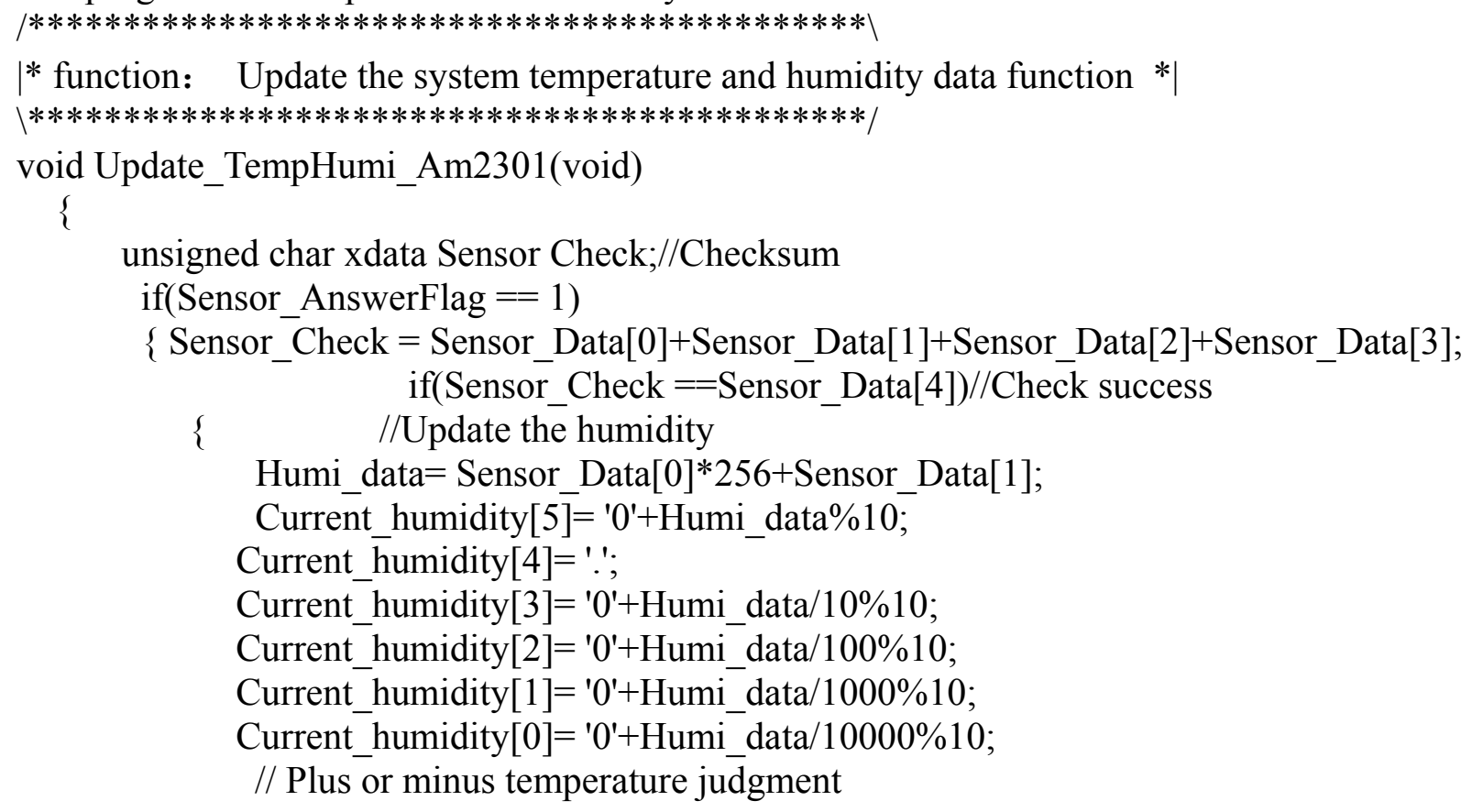




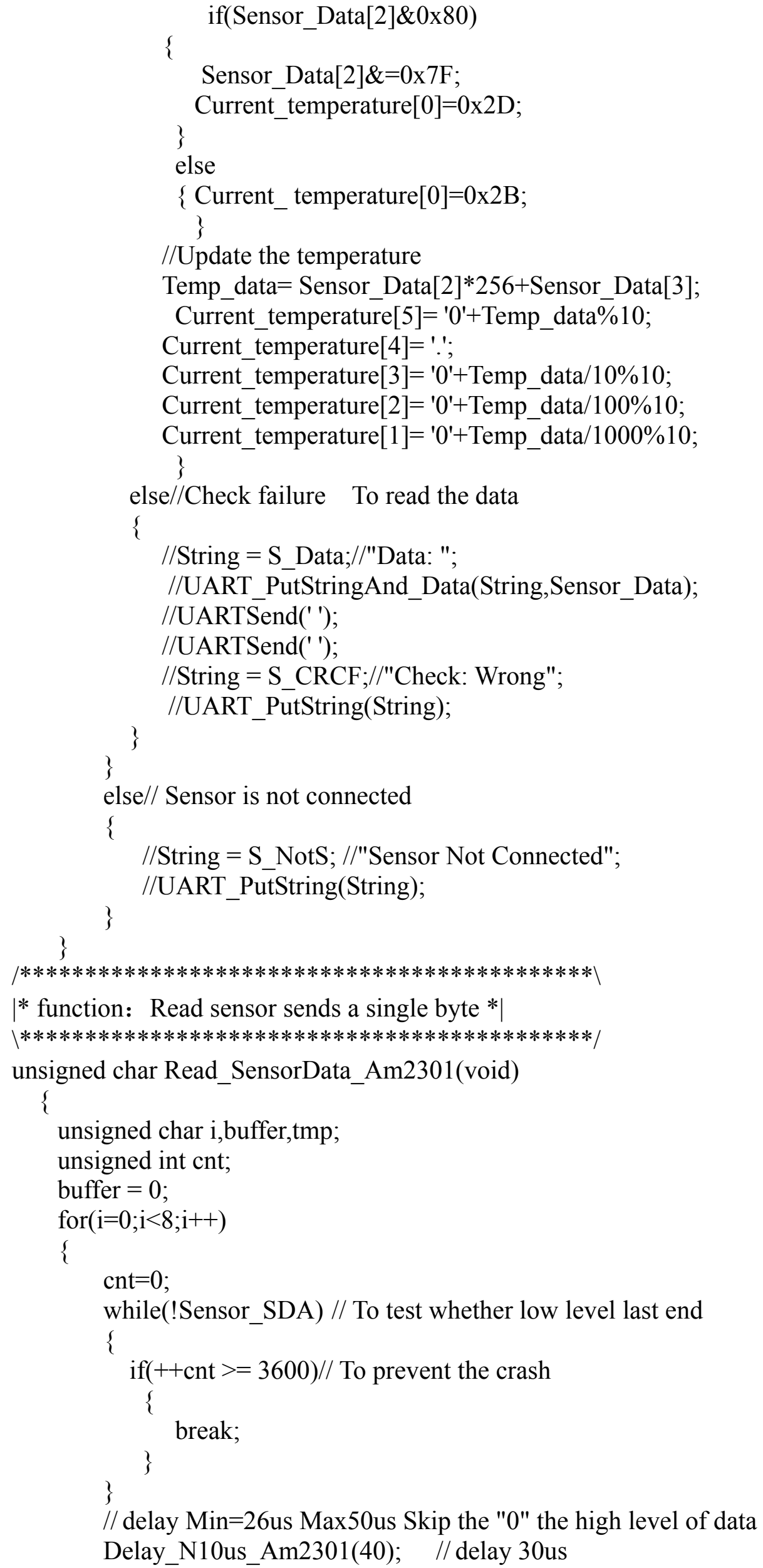

|* function: Read sensor sends a single byte $* \mid$

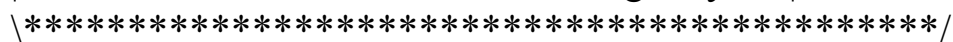

unsigned char Read_SensorData_Am2301(void) 


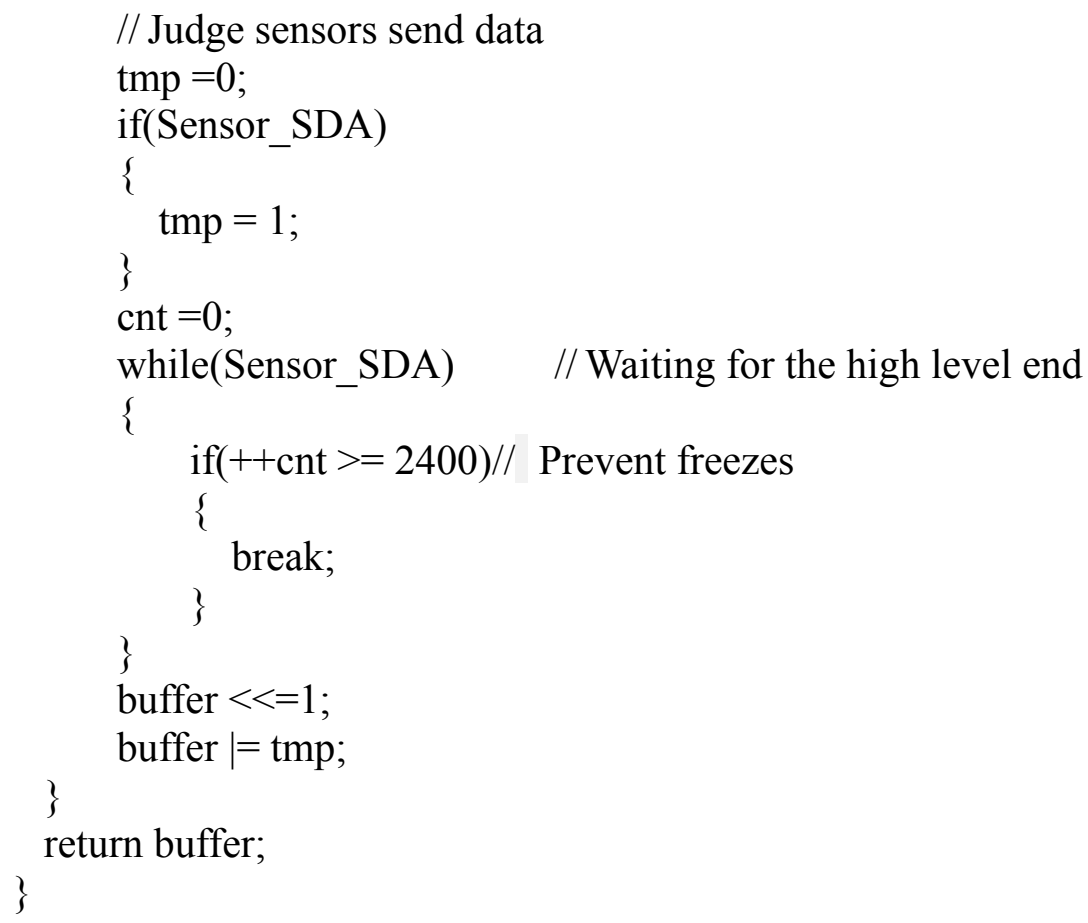

The test index is normal After the test.

\section{Conclusion}

Through reasonable design and a lot of experiments of the temperature and humidity sensor and vibration sensor interface circuit, the experiment parameters of coal mining blasting equipment library can reach the expected indicators, avoid safety accidents caused by explosives from the source. This system achieves the intelligent management for the storage and access for explosives and detonators, which can avoid the safety accidents from the greatest degree of caused by such sources.

\section{Acknowledgement}

In this paper, the research was sponsored by the Medical Science Research Projects in Henan Province (Project No. 201404065).

\section{Reference}

[1] Chenwei Cai, Haijian, Fan, Biyang Liu.Design and Application of Gate Control System Based on Fingerprint Identification Technology[J],Video Engineering. 2012.36(3) 128-130

[2] Siemens TC35/TC37 Hardware Interface [EB/OL].http//www.siemens.com/entry/cc/ en/.

[3] Wang YF. Xinxing Chuanganqi Jishu Ji Yingyong [M].Beijing: China metrology press, 2009:62

[4] Shuwen Guo, Harald Eriksen, Kimiko Childress etal. High temperature smart-cut SOI pressure sensor, Sensors And Actuators A:Physical. 2009,154(2)255 260..

[5] Li. Using single-chip microcomputer control cell phone text message [J]. Journal of electronic technology applications, 2002 (1) : 24-26. 